\title{
Dynamic Virtual Proteins: Visualization, Interaction and Collaboration in Virtual Reality
}

\author{
Jean-Marc Gauthier \\ Virtual Technology and Design \\ College of Art and \\ Architecture/University of Idaho, \\ Moscow, Idaho, USA, \\ gauthier@uidaho.edu
}

\author{
Jagdish Suresh Patel \\ Department of Biological Sciences \\ College of Science/University of \\ Idaho, Moscow, Idaho, USA, \\ jpatel@uidaho.edu
}

\author{
Ian McGrath \\ Institute for Modeling \\ Collaboration and \\ Innovation/University of Idaho \\ Moscow, Idaho, USA, \\ mcgr2676@vandals.uidaho.edu
}

\section{CCS CONCEPTS}

- CCS $\rightarrow$ Human-centered computing $\rightarrow$ Human interaction $(\mathrm{HCI}) \rightarrow$ Interaction paradigms

computer

\section{KEYWORDS}

Mixed Reality, Virtual Reality, Augmented Reality, Visualization, Interaction Design, User Interaction, User Experience, Usability, Protein Structure, Virtual Protein, Amino-acid, Bio-chemistry, Biology, Physics, Animation, Science, Education

\section{ACM Reference format:}

Jean-Marc Gauthier, Jagdish Suresh Patel and Ian McGrath. 2019. Dynamic Virtual Proteins: Visualization, Interaction and Collaboration in Virtual Reality. In Proceedings of VRST '19: 25th ACM Symposium on Virtual Reality Software and Technology (VRST '19), November 12-15, 2019, Parramatta, NSW, Australia. ACM, New York, NY, USA, 2 pages. https://doi.org/10.1145/3359996.3365050

\section{Introduction}

Current tools for visualizing and interacting with virtual proteins achieve a high level of complexity that creates a "communication barrier between structural biologists and other disciplines". Visualization tools may be difficult to use by a mixed audience of experts and non-experts. Challenges are not only technical but present cognition obstacles that may prevent people from sharing important knowledge between experts and non-experts. This can impact collaborations regarding engineering and decision making about new drugs, vaccines or treatments.

We selected and compared interactive visualization products that show proteins in virtual reality (VR) for education. The key elements observed for each software included: the choice of an open source platform to display the VR content, the type of

Permission to make digital or hard copies of part or all of this work for personal or classroom use is granted without fee provided that copies are not made or distributed for profit or commercial advantage and that copies bear this notice and the full citation on the first page. Copyrights for third-party components of this work must be honored. For all other uses, contact the owner/author(s).

VRST '19, November 12-15, 2019, Parramatta, NSW, Australia

(C) 2019 Copyright held by the owner/author(s).

ACM ISBN 978-1-4503-7001-1/19/11.

https://doi.org/10.1145/3359996.3365050 interactions that can be done with a virtual protein, the type of physics simulation, the use of virtual agents and the evaluation of the user experience in virtual reality. Some of the comparative products are VMD, Nanome from Nanome Technologies, iSciVIS and iSciLearn from Interactive Scientific and C4X Discoveries. As part of the development of the DVP, we propose a methodology of interaction design that combines human interactions and virtual agents assisting with the manipulation of a virtual protein. The proposed methodology closely follows the three-dimensional structure of a protein in order to design interactions that have strong theoretical foundations in biochemistry.

\section{Visualization of virtual proteins in VR}

In the case of the DVP, the challenge is to design a VR visualization and interaction system using user interface (UI) and user experience (UX) with a high degree of usability, clarity and speed. These elements will contribute towards fast interactions, ease of learning, and hopefully greater retention of information. We centered our research on a suite of tools for the design and evaluation of the UI and UX for an interactive visualization in VR. During the course of this project, we tested several virtual reality environments to evaluate the user interactions; offering feedback both for the user and for the designer of UI and UX in virtual reality.

\section{Collaboration with Virtual Agents}

The role of human spatial reasoning is "to improve transformation of space and the determination of when to pursue transformations using human decision making”. A concern of people manipulating virtual proteins is the accuracy of the location of atoms, amino acids and secondary structures in space with their respective forces. An increasing gap between the desired geo-localization of virtual protein elements by a VR team and the actual position of elements of a protein can generate errors. How do we know if a visualization is right or wrong in regards to space and time?

The "Design Interactions Feedback" is a proposed solution to correct the difference between the "desired" and "actual" position of elements for a virtual protein. Figure 1 shows the collaboration with virtual agents in the case of checking the location of elements of a virtual protein. A human participant, a 
virtual agent and the computer analytics collaborate following their interactions on separate tracks of the timeline. Virtual agents can relocate elements of a virtual protein at various stages of the visualization and interaction, making them valuable collaborators in the virtual world. In the case of manual operations, the participant can request for the virtual agent to intervene and correct possible imprecisions from their own manipulations.

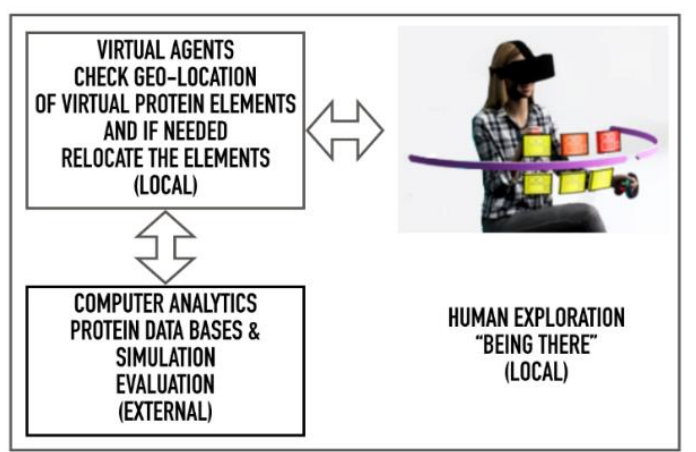

Figure 1: Diagram of collaboration between the human, virtual agents and computer analytics during the building of the alpha-helix structure.

\section{Interaction design methodology}

The DVP presents three levels of UI. The first is all manual, the second is collaboration with a virtual agent and the third is all automation. Figure 2 shows the process of "condensation" of five patterns used for the building of a secondary structure alphahelix into two patterns. The figure illustrates the first two possibilities: five manual human operations (blue arrows) or two operations in collaboration with virtual agents (orange arrows) which take over interaction patterns 3,4 and 5. The third level would not require a UI, as the virtual agents would be performing all tasks 'behind the scenes'. During a collaboration between a participant and a virtual agent, two interaction patterns appear on the user interface instead of five interaction patterns needed for a human working alone. The virtual agent will automatically execute the interaction patterns \#3, \#4 and \#5 that have been removed from the user interface.

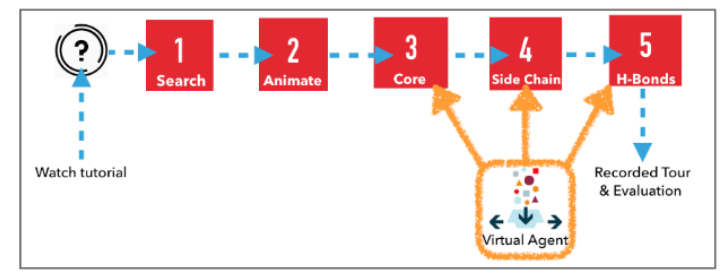

Figure 2: Top view, process of "condensation" of five patterns used by a human participant for the building of a secondary structure alpha-helix. The collaboration with a virtual agent reduces the user interface to 2 interaction patterns.

\section{Evaluation of a participant's interaction and collaboration using the DVP}

One of the goals of the DVP project is to collect scientific data for an evaluation of the user experience inside the virtual reality environment. The evaluation process leads to significant improvements of UI and UX. The DVP is currently being tested with high school and college students. Participants can use the DVP to visualize and interact with several virtual proteins in virtual reality. After generating a virtual protein in real time from the PDB, the participants have the possibility to manually manipulate the amino acids in order to recreate the secondary and tertiary structure of the virtual protein. They can choose to collaborate with a virtual agent that performs manipulations at the nanoscale level.

As a participant builds the dynamic tertiary structure of the virtual protein, a motion capture system records the navigation, gestures and activities of a participant inside the DVP. After completion of the tasks, the participant can visualize and take away a copy of his or her achieved goals and interaction session recorded as a set of key frames plotted inside a threedimensional heat map of the visualization of the virtual protein.

\section{ACKNOWLEDGMENTS}

The DVP project is funded by a NSF grant "RII Track-2 FEC: Using Biophysical Protein Models to Map Genetic Variation to Phenotypes".

Protein Models to Map Genetic Variation to Phenotypes". 